\title{
PROPRIÉTÉS UNIVERSELES DE CERTAINS SYSTÈMES DISCRETS DANS LE TEMPS
}

\author{
B. DERRIDA (*) \\ Institut Laue Langevin, 156X, 38042 Grenoble Cedex, France
}

\begin{abstract}
Résumé. -- Pour une classe assez large de systèmes à une dimension discrets dans le temps, il existe certaines propriétés universelles : l'ordre dans lequel les cycles limites stables apparaissent, la façon dont la longueur de ces cycles diverge pour certaines valeurs critiques. On peut montrer que le diagramme de bifurcations possède la propriété d'homothétie interne; cela permet de calculer par des méthodes de renormalisation les exposants universels qui décrivent le diagramme de bifurcations au voisinage des valeurs critiques et de démontrer que ce diagramme de bifurcations possède un ensemble non dénombrable de valeurs pour lesquelles il n'y a pas de cycle stable.
\end{abstract}

Abstract. - For a rather large class of uni-dimensional systems which are discrete in time, there exist certain universal properties : the order in which the stable limit cycles appear, how the length of these limit cycles diverges around some critical values. One can show that the bifurcation diagram possesses an internal similarity : this allows one to calculate with renormalization group methods the universal exponents which describe the behaviour of the bifurcation diagram around the critical values and to demonstrate that the bifurcation diagram has a non-countable set of points for which there is no stable limit cycle.

1. Ordre universel de l'ensemble des valeurs de bifurcation. - Quand on se donne un système discret [1] dans le temps :

$$
x_{t+1}=T\left(x_{t}\right)
$$

la question qui se pose est de savoir quel est le comportement quand $n \rightarrow \infty$ de $T^{n}\left(x_{t}\right)=x_{n+v}$. S'il existe une période $p$ stable, c'est-à-dire un ensemble de $p$ points $y_{1}, y_{2}, \ldots, y_{p}$ vérifiant :

$$
\begin{gathered}
y_{i+1}=T\left(y_{i}\right) ; \quad y_{1}=T\left(y_{p}\right) \\
-1<\left.\prod_{i=1}^{p} \frac{\mathrm{d} T}{\mathrm{~d} x}\right|_{y_{i}}<1
\end{gathered}
$$

alors tout un voisinage de ces points $y_{1}, y_{2}, \ldots, y_{p}$ finit par tomber sur cette période $p$.

Ainsi par exemple dans le cas de la figure $1 a$, $T(x)$ possède un point fixe stable, c'est-à-dire une période 1. Quand on déforme continûment $T(x)$, ce point fixe peut devenir instable et il apparait alors un cycle de période 2 (Fig. $1 b$ ) : quand $n \rightarrow \infty$ les 2 suites $T^{2 n}(x)$ et $T^{2 n+1}(x)$ convergent vers des limites différentes $y_{0}$ et $y_{1}$; après beaucoup d'itérations, on fait perpétuellement un mouvement d'aller et retour entre $y_{0}$ et $y_{1}$. On dit que la période 2 est

(*) Ce travail a été fait en collaboration avec Annie Gervois et Yves Pomeau. stable (Fig. 1b). Cette période 2 peut à son tour devenir instable et il apparaît alors une période 4 stable.
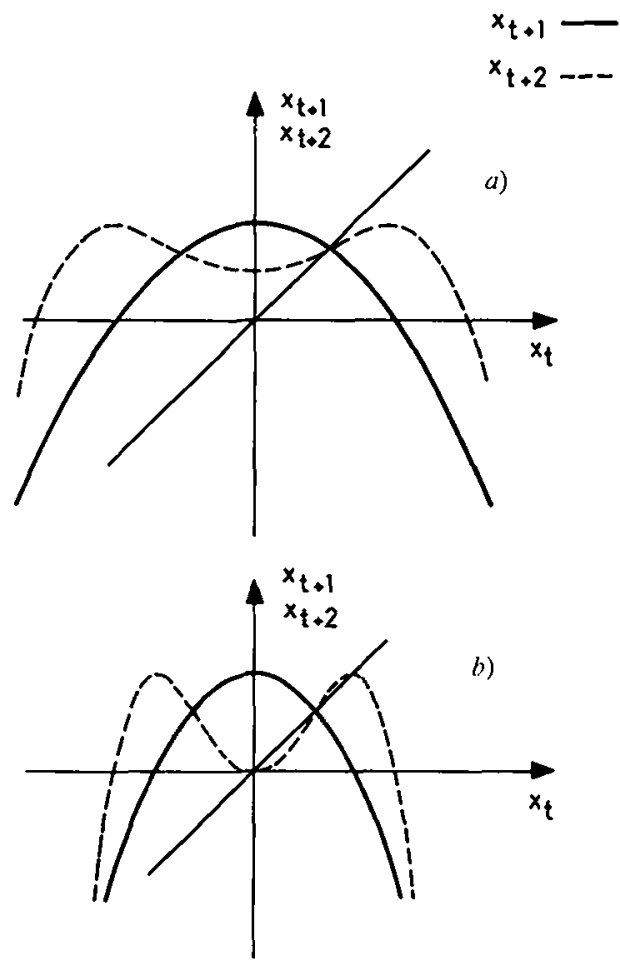

FIG. 1. - Sur la figure la, il n'y a qu'un point fixe stable. Quand on déforme $T(x)$, ce point fixe devient instable et il apparaît un cycle de période 2 (les 2 points du cycle sont points fixes de $T^{2}$ ) (Fig. 1b). 
Metropolis, Stein et Stein [2] ont décrit comment les périodes stables se succèdent quand on déforme continûment $T(x)$. Ils considèrent que $T$ a la forme :

$$
T_{\lambda}(x)=\lambda f(x)
$$

où $f(x)$ est une fonction ayant un seul maximum. $\lambda$ est le paramètre qui permet de déformer $T_{\lambda}(x)$; quand $\lambda$ varie, on peut représenter les intervalles de valeurs de $\lambda$ pour lesquelles une période est stable (Fig. 2).

\section{$\stackrel{1 \quad 24}{\longrightarrow}, 3+5 \stackrel{5}{\longrightarrow} \underset{+}{\longrightarrow} \longrightarrow$}

FIG. 2. - Longueur du cycle stable en fonction de $\lambda$. On n'a représenté icı que les zones de stabilité des périodes $\leqslant 5$.

Les valeurs de $\lambda$ qui limitent ces intervalles dépendent de la fonction $f$ mais d'après M.S.S. [2], pour une classe assez large de fonctions $f$ (à un seul maximum), ces intervalles sont rangés de la même manière. Autrement dit, indépendamment de la fonction $f$, quand $\lambda$ augmente, d'abord le point fixe est stable, puis la période 2 , puis la période 4 . Sur la figure 2 ne sont représentées que les périodes les plus basses. On peut montrer que le nombre de régions de stabilité d'une période $n$ croît comme $2^{n-1} / n$ quand $n \rightarrow \infty$ et il devient vite difficile de les représenter toutes.

Remarque. - Une période $n$ possède en général plusieurs zones de stabilité : ainsi par exemple la période 4 est stable 2 fois (Fig. 2). Ce qui distingue ces 2 zones de stabilité est la disposition des points de la période sur la droite réelle (Fig. 3).
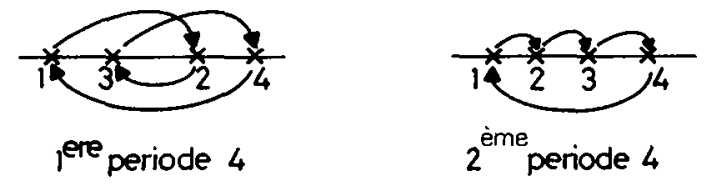

FIG. 3. - Formes des periodes 4

2. Homothétie interne [3]. - L'ordre [2] dans lequel les périodes stables se succèdent est assez compliqué. Cependant il possède une propriété simple d'homothétie interne.

Comme on peut le voir sur la figure 4 , si on choisit convenablement dans le diagramme de bifurcations (Fig. 4a) deux valeurs de $\lambda: \lambda_{2}$ et $\lambda_{2}^{\prime}$, on peut vérifier qu'entre $\lambda_{2}$ et $\lambda_{2}^{\prime}$ toutes les périodes stables sont paires et que les zones de stabilité de ces périodes (Fig. $4 b$ ) se succèdent dans le même ordre que dans le diagramme de bifurcations (Fig. 4a) à condition de multiplier les périodes par 2.

Il existe donc une fonction croissante $\mathfrak{H}_{2}$ telle que si $\Lambda=\mathscr{H}_{2}(\lambda)$, la période stable pour $\Lambda$ est 2 fois a)

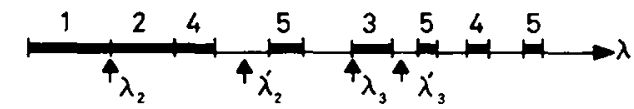

b)
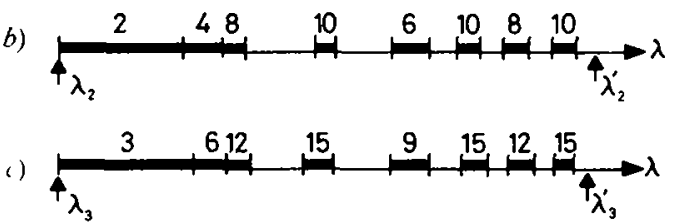

FIG. 4. - Si on regarde la structure du diagramme de bifurcations entre $\lambda_{2}$ et $\lambda_{2}$ sur la figure $4 a$, on obtient la figure $4 b$. Les périodes 6 , 8 et 10 de la figure $4 b$ existent effectivement sur la figure $4 a$ entre $\lambda_{2}$ et $\lambda_{2}^{\prime}$ mais n'ont pas été représentées. De même entre $\lambda_{3}$ et $\lambda_{3}^{\prime}$, la structure est donnée par la figure $4 c$.

plus longue que Ia période stable pour $\lambda$. Si $n(\lambda)$ est la longueur de la période stable de $T_{\lambda}$, on a :

$$
n\left(\mathcal{H}_{2}(\lambda)\right)=2 n(\lambda) \text {. }
$$

On peut trouver aussi 2 points $\lambda_{3}$ et $\lambda_{3}^{\prime}$ (Fig. 4a) entre lesquels le diagramme de bifurcations est reproduit dans sa totalité à condition de multiplier les périodes par 3 (Fig. 4c). Il existe donc une autre fonction croissante $\mathfrak{H}_{3}$ telle que :

$$
n\left(\mathscr{H}_{3}(\lambda)\right)=3 n(\lambda) .
$$

Plus généralement, à chaque période stable $p$, correspond une fonction $\mathscr{H}_{p}$ qui vérifie :

$$
n\left(\mathscr{H}_{p}(\lambda)\right)=p n(\lambda) .
$$

Le diagramme de bifurcations est donc similaire à certaines de ses parties.

3. Nombres universels et renormalisation [4], [5], [6]. - Nous avons vu que quand le point fixe se déstabilise, il apparaît une période 2 stable. D'après la propriété d'homothétie interne, quand la période 2 se déstabilise, il apparaît une période 4 stable et ainsi de suite : la période $2^{n}$ apparaît puis en se déstabilisant elle engendre une période $2^{n+1}$ stable.

Il existe une valeur $\lambda_{c}$ critique pour laquelle toutes ces périodes $2^{n}$ sont apparues et se sont déstabilisées (Fig. 5). On peut dire qu'au point $\lambda_{\mathrm{c}}$ la période stable est $2^{\infty}$ puisque cette valeur $\lambda_{c}$ est la limite des zones de stabilité des périodes $2^{n}$. On voit (Fig. 5) que la fonction $n(\lambda)$ (longueur de la période stable de la transformation $T_{\hat{\lambda}}$ ) diverge au point $\lambda_{\mathrm{c}}$.

Il se trouve que la fonction $n(\lambda)$ diverge avec une loi de puissance :

$$
n(\lambda) \sim\left(\lambda-\lambda_{\mathrm{c}}\right)^{\nu_{2}} \text { quand } \lambda \rightarrow \lambda_{\mathrm{c}}
$$

et on vérifie numériquement que l'exposant $v_{2}$ est le même pour toutes les fonctions $f$ (Eq. (1)) qui ont un comportement parabolique au voisinage de leur maximum. On peut mesurer numériquement cet exposant $v_{2}$ avec beaucoup de précision (en calculant numériquement toutes les valeurs de bifurcation). 


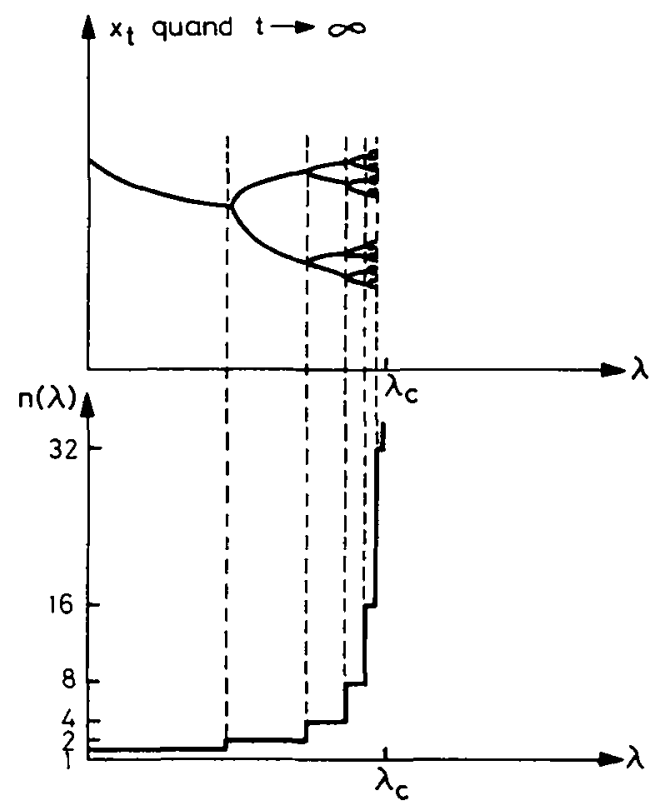

Fig. 5. - On représente les sauts de la fonction $n(\dot{\lambda})$ quand $\lambda$ augmente lors des premières bifurcations.

A partir de la relation (2), on voit que $\lambda_{c}$ est le point fixe de la fonction $\mathscr{K}_{2}$. En effet si

$$
\lambda_{c}=\mathscr{H}_{2}\left(\lambda_{c}\right)
$$

on a d'après (2) :

$$
n\left(\lambda_{c}\right)=2 n\left(\lambda_{c}\right)
$$

qui ne peut avoir comme solution que :

$$
n\left(\lambda_{\mathrm{c}}\right)=2^{\infty} \text {. }
$$

A partir de la connaissance de la fonction $\mathfrak{H}_{2}$, on peut calculer cet exposant par la relation :

$$
v_{2}=\frac{\ln 2}{\ln \left(\left.\frac{\mathrm{d} \mathscr{H}_{2}}{\mathrm{~d} \lambda}\right|_{i_{c}}\right)}
$$

En général, il n'est pas possible de connaitre la fonction $\mathscr{H}_{2}$ et donc de calculer exactement ces exposants. Cependant avec des formes approchées obtenues en cherchant quelle relation doit relier $\lambda$ et $\lambda$ pour que $T_{\lambda}$ et $T_{A}^{2}$ se ressemblent, on peut obtenir $v_{2}$ avec beaucoup de précision (Tableau I). Cette façon de procéder est calquée sur les méthodes de renorma-

\section{TABLEAU I}

Exposants pour les fonctions ayant un sommet parabolique

Méthode

Renormalisation la plus précise $v_{2} \quad v_{3}$ Valeur mesurée lisation dans l'espace direct utilisées dans la théorie des phénomènes critiques pour calculer les exposants.

Remarque. - Au voisinage de $\lambda_{\mathrm{c}}$ la fonction $n(\lambda)$ a des sauts et son comportement est de la forme :

$$
n(\lambda) \simeq\left(\lambda-\lambda_{c}\right)^{v_{2}} g_{2}\left(v_{2} \frac{\ln \left(\lambda-\lambda_{c}\right)}{\ln 2}\right)
$$

où la fonction $g_{2}$ est périodique : $g_{2}(z+1)=g_{2}(z)$.

De la même façon qu'au voisinage de $\lambda_{c}$, la fonction $n(\hat{\lambda})$ possède un exposant universel, $v_{2}$, au voisinage de la valeur $\lambda_{\mathrm{c}}^{\prime}$ point fixe de $\mathcal{H}_{3}$, la fonction $n(\lambda)$ a un comportement de la forme :

$$
n(\lambda) \simeq\left(\lambda-\lambda_{\mathrm{c}}^{\prime}\right)^{v_{3}} g_{3}\left(v_{3} \frac{\ln \left(\lambda-\lambda_{\mathrm{c}}^{\prime}\right)}{\ln 3}\right)
$$

et l'exposant $v_{3}$ est le même pour toutes les fonctions $f$ qui ont un sommet parabolique. Cet exposant peut aussi être mesuré et la meilleure valeur que l'on a calculée par des méthodes approchées est très proche de la valeur mesurée (Tableau I).

Remarque. - On peut généraliser ces comportements en mesurant et en calculant l'exposant $v_{p}$ qui correspond à l'homothétie interne $\mathfrak{H}_{p}$.

Remarque. - Dans certains cas, on peut trouver la forme exacte des fonctions $\mathscr{H}_{p}$ :

Si on considère la transformation $T$ qui dépend de 2 paramètres $(\lambda, r)$ définie par :

$$
\begin{aligned}
x<1, & T(x)=\lambda x \\
1<x<r, & T(x)=\lambda \\
x>r, & T(x)=\lambda(1+r-x) .
\end{aligned}
$$

On obtient par exemple :

$$
\begin{gathered}
\left(r^{\prime}, \lambda^{\prime}\right)=\mathscr{H}_{2}(r, \lambda) \Leftrightarrow\left\{\begin{array}{l}
\lambda=\lambda^{\prime 2} \\
r=\frac{\lambda^{\prime} r^{\prime}-1}{\lambda^{\prime}-r^{\prime}}
\end{array}\right. \\
\left(r^{\prime \prime}, \lambda^{\prime \prime}\right)=\mathscr{H}_{3}(r, \lambda) \Leftrightarrow\left\{\begin{array}{l}
\lambda=\lambda^{\prime \prime 3} \\
r=\frac{r^{\prime \prime} \lambda^{\prime \prime 2}-\left(\hat{\lambda}^{\prime \prime}+1\right)}{\lambda^{\prime \prime 2}-\left(\lambda^{\prime \prime}+1\right) r^{\prime \prime}} .
\end{array}\right.
\end{gathered}
$$

4. Ensemble des valeurs de $\lambda$ sans période stable. Si on considère l'ensemble $\varepsilon$ de toutes les valeurs de $\lambda$ pour lesquelles $T_{\lambda}$ n'a pas de période stable finie, cet ensemble s'applique dans lui-même par toutes opérations $\mathfrak{H e}_{p}$ :

$$
\forall p \quad \mathscr{H}_{p}(\mathcal{E}) \subset \mathcal{E} .
$$

Du simple fait que $\varepsilon$ est stable par 2 homothéties différentes (par exemple $\mathscr{H}_{2}$ et $\mathscr{H}_{3}$ ), cela permet de conclure que l'ensemble $\delta$ est non dénombrable [3]. C'est un ensemble de Cantor. Sauf pour certaines transformations $T_{\lambda}(x)$ bien particulières, on ne sait pas en général si cet ensemble est de mesure nulle : ainsi dans le cas de $T_{\lambda}(x)=\lambda x(1-x)$, on ne sait pas quelle est la mesure de $\varepsilon$. 


\section{Bibliographie}

[1] May, R. M., Nature 261 (1976) 10 (une large bibliographie).

[2] Metropolis, N., Stein, H. L. and Siein, P. R., Journal of Combinatorial Theory A 25 (1972).

[3] Derrida, B., Gervois, A. et Pomeau, Y., C.R. Hebd. Séan. Acad. Sci. 285 (1977) Série A43 et un article qui doit paraître dans Journal of Combinatorial Theory.
[4] Feigenbaum, M. J., Preprint LA-UR-77-1063 Quantitative universality for a class of non linear transformations.

[5] Coullet et Tresser, J. Physique Colloq. 39 (1978) C5-25.

[6] Derrma, B., Gervois, A. et Pomeau, Y., Un article en cours de rédaction. 